\title{
Voice problem in a patient with chronic renal failure
}

\author{
Effat Ahmed Zaky ${ }^{*}$ D, Haytham Mamdouh, Olivia Esmat and Zeinab Khalaf
}

\begin{abstract}
Background: Chronic kidney failure is an irreversible medical condition that impairs the kidney's ability to function. When CRF reaches a sophisticated stage, dangerous levels of fluid, electrolytes, and wastes can accumulate within the body. Dysphonia detected within the CRF patients was due to affection of the chronic kidney failure on the system and phonatory system. Patients with CRF treated by hemodialysis are exposed to continuous pulmonary insults of multifactorial origin: Fluid retention predisposes them to pulmonary edema which occurs more frequently within the presence of concomitant cardiovascular disease. Also, the spirit of the kidney failure patients can induce psychogenic dysphonia. The aim of this work is to see and analyzed voice problems in patients with chronic kidney failure to ascertain baseline data about the scale and distribution of the probable voice disorder in these patients for early detection and proper management.

Results: The results obtained from this study showed that there have been statistically significant differences between chronic kidney failure patients $\mathrm{G} 1$ and control G2 regarding first harmonic, jitter \%, shimmer $\mathrm{dB}$ and noise harmonic ratio dB, presence of dysphonia, and also the total score of VHI. The results of the study revealed statistical correlation between the quantity of years of hemodialysis and total acoustic measures.

Conclusion: The results of our study revealed that subjects with chronic failure exhibit a clinical evidence of voice disorders and proving that there is interplay of different body systems and the larynx. The voice problems can vary between CRF patients depending on duration of hemodialysis and leading causes of chronic kidney failure.
\end{abstract}

Keywords: Dysphonia, Chronic failure, Jitter, Shimmer

\section{Background}

Hemodialysis is the inevitable treatment procedure for end-stage renal disease. Hemodialysis aims to get rid of the surplus fluids and toxins and improves chemical equilibrium. Voice production involves price coordination between the central system and peripheral phonatory organs. These features of end-stage renal disease can cause the change of voice attributable to decreased lung function and edema of the vocal folds. If hemodialysis is successful, lung functions improved, and also the volume of the vocal cord is decreased [1]. Most patients with end-stage renal disease after $3-5 \mathrm{~h}$ of hemodialysis, experience general weakness, fatigue, and

* Correspondence: dr_efat_am@yahoo.com

Department of Otolaryngology, Phoniatrics Unit, Minia Faculty of Medicine, Minia University, 31 El Gomhoria Street, El, Minia 61111, Egypt voice change that lasts for some hours. Change of voice has been reported in $24-60 \%$ of the patients with endstage renal disease after completion of every hemodialysis session [2]. Belafsky et al. [3] found that excessive fluid within the superficial lamina propria of the vocal cord "Reinke's space" in a very patient with end-stage renal disease. Moreover, pulmonary calcification which is common in chronic dialysis patients and pulmonary dysfunction, of these factors can cause increased muscle tension within the vocal tract and consequently induce dysphonia. Also, the spirit of the kidney failure patients can induce a psychogenic dysphonia.

This work aims to see and analyzed voice problems in patients with chronic kidney failure to ascertain baseline data about the scale and distribution of the probable 
voice disorder in these patients for early detection and proper management.

\section{Methods}

This prospective study was conducted in our Otolaryngology-Head and Neck Surgery Department and Phoniatrics Unit, from Jan. 2016 to Feb. 2019. This study included 110 individuals, age ranging from 25 to 70 years. The (study group) (G1) included 54 patients with chronic renal failure (CRF). The study group was compared to another group (control group) (G2), which included 56 individuals with no renal problems. Both of the study and control groups were statistically matched in the comparative data age and sex distribution. G1 included 54 patients diagnosed as chronic renal failure. They were 38 males (70.3\%) and 16 females (29.6\%), with a range of age ranging from 25 to 70 years old, referred to our Phoniatrics Unit from the Nephrology Unit. The subject-inclusion criteria for the clinical group were patients with chronic renal failure as diagnosed by experienced nephrologists, undergoing hemodialysis three times per week for more than 1 year. The exclusion criteria were history of voice misuse or abuse and history of laryngeal surgery. G2 included 56 normal individuals. They were 40 males $(71.4 \%)$ and 16 females (29.5\%) with a range of age of 25 years to age 70 years, they were collected randomly from the relatives of patients who frequently attend to outpatient clinic of internal medicine, relatives also of the children who are following up at the Phoniatrics Unit.

Each individual of both groups were subjected to the following protocols of assessment. All the patients and individuals of the control group signed consent to be enrolled in the study.

[A] The full voice evaluation protocol in the Phoniatrics Unit [4].

[B] Arabic Voice Handicap Index (VHI): The Voice Handicap Index (VHI) was filled by all patients if they were literate; illiterate patients were asked the questions of $\mathrm{VHI}$ and the researcher filled the answers. The Arabic version of the VHI was used (Appendix), it consists of 30 items selfadministrated questionnaire that asked the patients to describe their voice and quantify the functional, physical, and emotional impacts of a voice disorder on a patient's quality of life [5].

[C] Acoustic measurements: All groups were analyzed by a modified GRBAS scale 4; those with a rating of more than 0 were excluded. Speech Studio is a Windows-based, real-time, multimedia, speech display and replay system linked to statistical analysis programs for the assessment of speech production and perception. Speech Studio facilitates work with real, continuous speech.

[D] Kidney functions: Chronic renal failure is defined as either kidney damage or glomerular filtration rate less than $15 \mathrm{~mL} / \mathrm{min} / 1.73 \mathrm{~m}^{2}$ for 3 months or more. This is invariably a progressive process that results in end-stage renal disease. Serum creatinine is commonly used to estimate creatinine clearance but is a poor predictor of glomerular filtration rate, as it may be influenced in unpredictable ways as assay techniques, endogenous, and exogenous substances, renal tubular handling of creatinine, and other factors (age, sex, body weight, muscle mass, diet, drugs) [6]. The glomerular filtration rate is the "gold standard" for determining kidney function, but its measurement remains cumbersome. For practical purposes, calculated creatinine clearance is used as a correlate of glomerular filtration rate and is commonly estimated by using CKD-EPI Creatinine 2009 equation which estimated from serum creatinine, age, sex, and race.

Expressed as a single equation: eGFR $=141 \times$ $\min (\mathrm{Scr} / \kappa, 1) \alpha \times \max (\mathrm{Scr} / \kappa, 1)-1.209 \times 0.993 \mathrm{Age} \times$ 1.018 [if female] $\times 1.159$ [if black].

SCR is serum creatinine $(\mathrm{mg} / \mathrm{dL}), \kappa$ is 0.7 for females and 0.9 for males, $\alpha$ is -0.329 for females and -0.411 for males, min indicates the minimum of $\mathrm{Scr} / \kappa$ or 1 , and max indicates the maximum of $\mathrm{Scr} / \kappa$ or 1 .

The data were analyzed using the Statistical Package for the Social Sciences (SPSS) Version 22. The quantitative data were presented by mean, median, and standard deviation while the qualitative data were presented by frequency distribution. A Pearson product-moment correlation test was used to determine the relationship between nasal and laryngeal lesions. The probability of less than 0.05 was used as a cut-off point for all significant tests.

\section{Results}

There were non-statistically significant differences between the patient group (GI) and the control group (GII) regarding the age and sex $(p=0.01)$ (Table 1$)$.

This study carried on 56 patients suffering from CRF with the mean years of hemodialysis $7.16 \pm 4.70$ and a range (1-18), 17 (31.5\%) of them were diabetics, 14 (25.9\%) were hypertensive, 11 (20.4\%) were with repeated kidney stones, 1 (1.9\%) with repeated urinary infection and were on narcotics, and 9 (16.7\%) were diabetic and hypertensive.

There were statistically significant differences between CRF patients G1 and control G2 regarding fundamental frequency $\mathrm{Hz}(p=0.001 *)$. There were statistically highly significant differences in both groups regarding jitter \% 
Table 1 Sociodemographic data in case and control groups

\begin{tabular}{llll}
\hline Socio-demographic characteristics & Cases $(\boldsymbol{N}=\mathbf{5 4 )}$ & Control $(\boldsymbol{N}=\mathbf{5 6})$ & $\boldsymbol{t}(\mathbf{d f}), \boldsymbol{p}$ value \\
\hline Age (years) & $46.66 \pm 12.65$ & $45.55 \pm 11.05$ & $4.89(104.93), 0.01$ \\
Sex & & & $5.84(1), 0.01$ \\
$\quad$ Male & $38(70.3 \%)$ & $40(71.4 \%)$ & $16(29.5 \%)$ \\
$\quad$ Female & $16(29.6 \%)$ & & \\
\hline
\end{tabular}

Non-significant $(p>0.05)$, significant $(p<0.05)$, highly significant $(p<0.001)$

$\left(p<0.001^{*}\right)$. There were statistically significant differences between the two groups regarding shimmer $\mathrm{dB}(p$ $=0.03 *)$. There were statistically significant differences between both groups regarding noise harmonic ratio $\mathrm{dB}$ $\left(p=0.001^{*}\right)$ (Table 2).

There were highly statistically significant differences between CRF patients G1 and control G2 regarding the grade of dysphonia ( $p<0.001)$, in G1, $20(37.03 \%)$ had dysphonia, 13 (24.1\%) of them had grad 1 of dysphonia, 7 (13\%) of them had grad 2 of dysphonia. While in G2, 4 (7.14) had dysphonia, 4 (7.1\%) of them had grade 1 of dysphonia, $0(0 \%)$ of them had grade 2 of dysphonia. As regard to character of dysphonia, there were statistically significant differences between both groups $(p<0.001)$ regarding strained, leaky, and strained and leaky, in G1, 12 (22.2\%) of them had strained dysphonia, 5 (9.3\%) of them had leaky dysphonia and 3 (5.6\%) of them had strained and leaky dysphonia. As regard to the pitch, there were highly statistically significant differences between both groups $(p<0.001)$; in G1, $27(50 \%)$ of them had high pitched voice while $1(1.9 \%)$ of them had low pitched voice. In G2, 1 (1.8\%) had high-pitched voice and 1 (1.8) had low-pitched voice. As regard loudness, there were statistical differences between both groups $(p<0.001)$, in G, 39 (72.2\%) had soft loudness and $15(27.8 \%)$ of them had decreased loudness while in G2, 55 (98.2) of them had soft loudness and 1 (1.8\%) of them had decreased loudness. As regard associated laryngeal functions, there were statistical differences between both groups, in G1, 20 (37\%) of them were affected and 34 (63\%) of them were not affected while in G2, 1 (1.8\%) of them were affected and $55(98.2 \%)$ of them were not affected (Table 3$)$.

There were statistically significant differences observed between CRF patients G1 and control G2 regarding the VHI functional handicap $(p<0.01)$. There were statistically significant differences observed between CRF patients G1 and control G2 regarding the VHI physical handicap $(p<0.01)$. There were statistically significant differences observed between CRF patients G1 and control $\mathrm{G} 2$ regarding the VHI emotional handicap $(p<0.01)$. There were statistically significant differences observed between CRF patients G1 and control G2 regarding the total score of VHI $(p<0.01)$ (Table 4).

There were statistically significant differences observed between CRF patients $\mathrm{G} 1$ and control G2 regarding the endoscopic finding $(p=0.03)$. In $\mathrm{G} 1,35(64.8 \%)$ of them were normal, $6(11.1 \%)$ of them have mild congestion, 4 (7.3\%) of them had bilateral vocal fold hypertrophy, 1 (1.9\%) of them has bilateral vocal fold nodules, 7 (13\%) have mild congestion and vocal fold hypertrophy, and 1 $(1.9 \%)$ have congestion and vocal fold nodules. In G2, 51 (91.1\%) of them were normal, 3 (5.3\%) of them had mild congestion, $1(1.8 \%)$ of them had bilateral vocal fold hypertrophy, $0(0 \%)$ of them had bilateral vocal fold nodules, $1(1.8 \%)$ of them had mild congestion and vocal fold hypertrophy, and $0(0 \%)$ had mild congestion and vocal fold nodules (Table 5).

This study was carried on 54 CRF patients, with a mean of serum creatinine $4.04 \pm 1.13$ and a range (2.1-6.3), mean of serum urea $83.70 \pm 12.92$ and a range (65-119), and mean of GFR $12.27 \pm 8.81$ and a range (9.5-16.2) (Table 6).

The results of the study revealed a statistically significant positive correlation between the number of years of hemodialysis and total acoustic measures, total FO, total jitter, total shimmer, and total NHR (Table 7).

There was a statistically positive correlation between diabetes and hypertension as a cause of CRF and total VHI, with diabetes and hypertension. There was a statistically positive correlation between diabetes and hypertension as a cause of CRF and dysphonia (Tables 7 and 8).

Table 2 Total acoustic measures in cases and control groups

\begin{tabular}{llll}
\hline & Cases $(\boldsymbol{N}=\mathbf{5 4})$ & Control $(\boldsymbol{N}=\mathbf{5 6})$ & $\boldsymbol{t}(\mathbf{d f}), \boldsymbol{p}$ value \\
\hline Total FOA $($ mean $\pm \mathrm{sd})$ & $202.28 \pm 51.68$ & $167.68 \pm 58.52$ & $3.28(107.18), 0.001^{*}$ \\
Total jitter $($ mean $\pm \mathrm{sd})$ & $1.45 \pm 1.09$ & $0.80 \pm 0.40$ & $4.11(66.99),<0.001^{*}$ \\
Total shimmer $($ mean $\pm \mathrm{sd})$ & $1.15 \pm 0.73$ & $0.89 \pm 0.58$ & $2.08(101.10), 0.03^{*}$ \\
Total HNR $($ mean $\pm \mathrm{sd})$ & $19.38 \pm 3.33$ & $16.08 \pm 5.03$ & $3.30(108), 0.001^{*}$ \\
\hline
\end{tabular}

Non-significant $(p>0.05)$, significant $(p<0.05)$, highly significant $(p<0.001)$ 
Table 3 Auditory perceptual assessment in case and control groups

\begin{tabular}{|c|c|c|c|}
\hline Auditory perceptual assessment & Cases $(N=54)$ & Control $(N=56)$ & $x^{2}(\mathrm{df})^{*}, p$ value \\
\hline \multicolumn{4}{|l|}{ Dysphonia $N(\%)$} \\
\hline Number & $20(37.03 \%)$ & $4(7.14)$ & $15.50(2),<0.001^{*}$ \\
\hline Grade 1 & $13(24.1 \%)$ & $4(7.1 \%)$ & \\
\hline Grade 2 & $7(13 \%)$ & $0(0 \%)$ & \\
\hline \multicolumn{4}{|l|}{ Character $N(\%)$} \\
\hline Free & $34(63 \%)$ & $52(92.9 \%)$ & $18.10(3)_{,}<0.001^{*}$ \\
\hline Strained & $12(22.2 \%)$ & $0(0 \%)$ & \\
\hline Leaky & $5(9.3 \%)$ & $6(10.6 \%)$ & \\
\hline Strained and leaky & $3(5.6 \%)$ & $0(0 \%)$ & \\
\hline Pitch $N$ (\%) & & & $33.91(2),<0.001^{*}$ \\
\hline Normal & $26(48.1 \%)$ & $54(96.4 \%)$ & \\
\hline Increased & $27(50 \%)$ & $1(1.8 \%)$ & \\
\hline Decreased & $1(1.9 \%)$ & $1(1.8 \%)$ & \\
\hline Register $N(\%)$ & & & $0.001(1), 0.9$ \\
\hline Modal & $53(98.1 \%)$ & 55 (98.2\%) & \\
\hline Vocal fry & $1(1.9 \%)$ & $1(1.8 \%)$ & \\
\hline Loudness $N(\%)$ & & & $14.94(1)_{1}<0.001^{*}$ \\
\hline Soft & 39 (72.2\%) & $55(98.2 \%)$ & \\
\hline Decreased loudness & $15(27.8 \%)$ & $1(1.8 \%)$ & \\
\hline \multicolumn{4}{|l|}{ Glottal attack } \\
\hline Hard & $0(0 \%)$ & $0(0 \%)$ & \\
\hline Not hard & $54(100 \%)$ & $56(100 \%)$ & \\
\hline Associated laryngeal functions $N(\%)$ & & & $22.11(1)_{1}<0.001^{*}$ \\
\hline Affected & $20(37 \%)$ & $1(1.8 \%)$ & \\
\hline Not affected & $34(63 \%)$ & $55(98.2 \%)$ & \\
\hline
\end{tabular}

Non-significant $(p>0.05)$, significant $(p<0.05)$, highly significant $(p<0.001)$

\section{Discussion}

Change of voice related to chronic kidney disease has attracted good attention for clinical researches; however, change of voice may be a crucial source of hysteria for the patients and their relatives [7]. CRF affects the voice as a side effect of hemodialysis and medications or associated concurrent diseases [8]. This study aimed to work out and analyze voice problems in patients of chronic kidney disease G1 compared with normal persons G2 to early detection and proper management. Our study consisted of 54 patients with CRF diagnosed by experienced nephrologist: undergoing hemodialysis 3 times per week for over 1 year and by renal functions include (serum urea, serum creatinine, and glomerular filtration rate) and 56 normal individual, each individual of both groups were subjected to a full voice evaluation which incorporates elementary diagnostic procedures, clinical diagnostic aids, VHI, and acoustic measurements.

We observed a highly statistical significant difference between GI and GII, regarding the basic frequency and also the pitch. These results may be explained by several factors as removal of excessive fluids and uremic toxins from the body by the hemodialysis result in good improvement of the intrinsic laryngeal muscles especially

Table 4 Parameters of voice handicapped index in case and control groups

\begin{tabular}{llll}
\hline & Cases $(\boldsymbol{N}=\mathbf{5 4})$ & Control $(\boldsymbol{N}=\mathbf{5 6})$ & $\boldsymbol{t}$ (df), $\boldsymbol{p}$ value \\
\hline Functional (mean $\pm \mathrm{sd})$ & $1.53 \pm 2.25$ & $0.23 \pm 0.76$ & $4.03(64.58),<0.01^{*}$ \\
Physical (mean $\pm \mathrm{sd})$ & $1.46 \pm 2.16$ & $0.03 \pm 0.18$ & $4.81(53.76),<0.01^{*}$ \\
Emotional (mean $\pm \mathrm{sd})$ & $0.92 \pm 1.39$ & $0.10 \pm 0.36$ & $4.16(59.96),<0.01^{*}$ \\
Total VHI & $3.79 \pm 5.14$ & $0.37 \pm 1.25$ & $4.75(59.11),<0.01^{*}$ \\
\hline
\end{tabular}

Non-significant $(p>0.05)$, significant $(p<0.05)$, highly significant $(p<0.001)$ 
Table 5 Endoscopic findings case and control groups

\begin{tabular}{llll}
\hline Endoscopic finding & Cases $(\boldsymbol{N}=\mathbf{5 4 )}$ & Control $(\boldsymbol{N}=\mathbf{5 6})$ & $\boldsymbol{X} \mathbf{2}(\mathbf{D F})^{*}, \boldsymbol{p}$ value \\
\hline Normal & $35(64.8 \%)$ & $51(91.1 \%)$ & $12.24(5), 0.03^{*}$ \\
Mild congestion & $6(11.1 \%)$ & $3(5.3 \%)$ & $1(1.8 \%)$ \\
Vocal fold hypertrophy & $4(7.3 \%)$ & $0(0 \%)$ \\
Nodules & $1(1.9 \%)$ & $1(1.8 \%)$ \\
Congestion and vocal fold hypertrophy & $7(13 \%)$ & $0(0 \%)$ \\
Congestion and nodule & $1(1.9 \%)$ & \\
\hline
\end{tabular}

Non-significant $(p>0.05)$, significant $(p<0.05)$, highly significant $(p<0.001)$

pitch rising muscles, additionally, to the advance occurring of the lung functions, the dehydration condition occurring with hemodialysis could also causing an increase of the basic frequency, because either hemodialysis or peritoneal dialysis allowing an outsized volume decrease in body hydration (ultrafiltration), briefly time (3-4h), and a couple of to three times per week which strongly affect the respiratory and phonatory systems and contributing the rise of the pitch as a result of decrease in mass. The fear and anxiety anticipated by the dialysis session within these patients also may result in a rise in their pitch although these patients are already in the state of chronic stress. Many studies are in line with our results as Venkata et al. [9] who reported that chronic hemodialysis patients may have a decrease within the plica vocalis thickness. This decrease within the plica vocalis thickness could be the result from dehydration. Kumar and Bhat [10] also exhibited in their study the next first harmonic in $54 \%$ of their chronic kidney disease patients compared with normal subjects. Hemler et al. [11] sought that superficial drying of excised plica vocalis mucosa increased its viscosity making the vocal folds more stiffened which lead to a big increase in phonation threshold pressure which successively result in a rise of the basic frequency. Chen et al. [12] reported that the first harmonic showed an identical increase in patients with chronic kidney disease; thanks to changes within the biochemicals parameters that affected muscle performance and also the first harmonic is that the results of harmony between airflow and laryngeal muscle biomechanics.

Table 6 Descriptive statistics of renal function parameters in cases

Cases $(N=54)$

\begin{tabular}{l}
\hline Serum creatinine \\
$\quad$ Range (mean $\pm \mathrm{sd})$ \\
Serum urea \\
$\quad$ Range (mean $\pm \mathrm{sd}$ ) \\
GFR \\
$\quad$ (6.1-6.3) $4.04 \pm 1.13$ \\
Range (mean $\pm \mathrm{sd}$ )
\end{tabular}

Normal serum creatinine level, $0.5-1.2 \mathrm{mg} / \mathrm{dl}$

Normal urea nitrogen blood, $7-20 \mathrm{mg} / \mathrm{dl}$

Normal GFR, $116-57 \mathrm{~mL} / \mathrm{min} / 1.73 \mathrm{~m}^{2}$
Therefore, Nesic et al. [13] exploring the link between the psychosomatic state of patients on dialysis and also the acoustic parameters of the vowel "a" and that they found that stress effects on respiration and muscle tension and possibly to affect phonation and articulation. The results showed that within the period before dialysis in $60 \%$ of his patients, the basic frequency was greater, duration was longer and intensity was unchanged, but the amount before the treatment was related to special, anticipatory stress that induced greater first harmonic and longer duration of the pronunciation of the vowel "a." Ori et al. [2] examined the plica vocalis thickness of 38 vocal folds for 16 patients post-dialysis. They concluded that "the decrease in plica vocalis thickness was by $10.9 \%$, which may result from the dehydration." But these results are not in agreement with the study by Eman [14] who reported that male patients with CRF exhibited significantly increase first harmonic compared with normal male individuals. However, there was no significant difference in the first harmonic within the total group and within the female subgroup. But she explained that the decreased serum testosterone level in male patients with CRF.

There were highly statistically significant differences between both groups regarding the jitter \%, shimmer $d B$, and noise harmonic ratio $\mathrm{dB}$. We found that jitter-related measures or relative-average perturbation (RAP) and shimmer-related measures were found to be higher in persons with chronic kidney disease compared with normal subjects, thanks to decreased phonatory control leading to irregular vibration of the vocal folds. That decrease in phonatory control may be, thanks to the negative fluid balance effect of chronic hemodialysis which affecting on the laryngeal muscles. We also found that jitter and shimmer values might be also laid low with dehydration state and this in agreement with Maria and Kenneth [15] who suggested that both values are significantly increased in dehydration condition which was defined as "fasting for $14 \mathrm{~h}$, or not ingesting foods or liquids for this era and retain again to their normal values within the rehydration condition which was defined as ingesting $1 \mathrm{~L}$ of water in $20 \mathrm{~min}$," supporting the hypothesis that systemic hydration positively regulates plica vocalis biomechanical properties by increasing vocal tissue viscosity. These results 
Table 7 Correlation between years of hemodialysis and total acoustic measures

\begin{tabular}{llllll}
\hline Pearson correlation & & Total FOA & Total jitter & Total shimmer & Total HNR \\
\hline Years of HD & $r$ & 0.685 & 0.640 & 0.691 & 0.597 \\
& $p$ value & $0.04^{*}$ & $0.02^{*}$ & $0.01^{*}$ & $0.01^{*}$ \\
\hline
\end{tabular}

were in line with many studies as Eman [14] who founded that $42 \%$ of the patients with chronic kidney disease presented with higher shimmer values and NHR than normal persons caused by a protracted glottic opening lead to excessive airflow that perceived as a periodic noise. This turbulence of noise has no harmonics, Kumar and Bhat [10] also reported a rise in shimmer in both male and feminine patients with CRF, thanks to the lack of the participants to take care of a continuing intensity during the phonation of /a/ while NHR was not assessed in their study. Controversy to the study by Unver et al. [16] who revealed a big decrease in NHR and that they contributed that to the dehydration occurred in hemodialysis patients and losing of the hemostatic mechanisms that regulate the hydration of important airway tissues (including the larynx) despite systemic hydration challenges, such mechanisms preserve effective mucociliary clearance, airway tissue compliance.

We found that there have been statistically significant differences between CRF patients G1 and control G2 regarding the presence of dysphonia. We found that chronic kidney failure patients were more likely to own a change of voice because dysphonia originates mainly from the weak pulmonary support of the voice. Patients with endstage renal disease treated by hemodialysis are exposed to continuous pulmonary insults of multifactorial origin: Fluid retention predisposes them to pulmonary edema, which occurs more within the presence of concomitant cardiovascular disease. Also, pulmonary calcification which is common in chronic dialysis patients and pulmonary dysfunction can cause increased muscle tension within the vocal tract and consequently induce dysphonia. Pulmonary functions in kidney failure patients below normal subjects and those they compensate by increasing their expiratory pressure and expiratory pulmonary effort. Within the future, it ends up in increased expiratory effort during phonation which successively increases the stress within the laryngeal muscles and leads to hyperfunctional dysphonia. Another important factor also is the noise within the hemodialysis unit and high-frequency attenuation by the airflow masks; all of those factors make the patients increasing their vocal efforts and inducing voice

Table 8 Correlation between diabetes and hypertension as Causes of renal failure and APA

\begin{tabular}{llll}
\hline Spearman correlation & Diabetes & Hypertension \\
\hline Dysphonia & $r$ & 0.282 & 0.49 \\
& $p$ value & $0.03^{*}$ & $0.03^{*}$ \\
\hline
\end{tabular}

changing. Also, the emotion of the kidney failure patients can induce a psychogenic dysphonia. Ori et al. [2], who reported that $24-60 \%$ of the patients with ESRD (end-stage renal disease)is more likely to be presented with postdialysis dysphonia.

There were statistically significant differences between both groups regarding the character of the voice. The looks of strained quality may be explained by the dehydration state occurring during hemodialysis cause increase PTP (phonation threshold pressure) which successively increase the vocal effort and end in a tense voice that is perceived as strained quality. The voice is perceived as leaky additionally to strain when the strained voice with increased glottis and supraglottic activity and when vocal folds cannot come perfectly together from partial nerve input loss. Dysphonia may cause pain or a strained feeling when trying to talk normally. Change of voice may be caused by anything that interferes with the conventional vibration of the vocal folds, like swelling or inflammation or affection on the biomechanical of the vocal tissues [17].

In our study, there have been statistically significant differences observed between CRF patients G1 and control G2 regarding the endoscopic findings. The presence of vocal folds hypertrophy may well be explained by that the patient tried to atone for the high vocal effort required for phonation by glottic and supraglottic hyperactivity which explains the looks of strained quality. The structural changes (vocal folds congestion and plica vocalis nodules) may well be attributed to hyperfunctional elements as voice misuse or abuse.

We observed statistically significant differences observed between CRF patients G1 and control G2 regarding the VHI functional handicap. These results may well be attributed thereto; the results were explained by the dysphonia which can be a reliable reflection of the degree of voice handicap. The more severe is the degree of dysphonia, the tougher for people to listen to, the more restriction in joining a conversation with the resultant emotional effects on the individual himself. Voice Handicapped Index (VHI) could be a useful measure that would help the individual and therefore the clinician to assess the degree of disability caused by voice disorders. CRF could be a disease that hurts the patient's communication with other individuals.

We observed no statistical correlation between the overall score of VHI and GFR and no statistical correlation between GFR and acoustic parameters, respectively with first harmonic, jitter, shimmer, and noise-harmonic 
ratio. This might be attributed thereto the voice changes occurring with kidney failure thanks to pulmonary insults like pulmonary muscle weakness, pulmonary calcifications, and pulmonary edema which affect the intrinsic and extrinsic laryngeal muscles. Therefore, the GFR become unuseful to administer us a plan about the extension of those changes but we observed that there have been a statistically significant direct correlation between the number of years of hemodialysis or in other words, several years of kidney failure and total acoustic measures within the sort of first harmonic, jitter, shimmer, and noise harmonic ratio and that we found that the weakness of the respiratory muscles and therefore the affection of whole pulmonary system increase by the duration of the hemodialysis and become more severe. Many studies are in line with our results as Moinard and Guenard [18] who texted that "the low incidence of the respiratory complications in patients with long-standing severe nephropathy not treated with hemodialysis and in patients enrolled on dialysis programs for a brief period of your time (less than 12 months) suggests that the pathogenetic factor(s) involved in pulmonary abnormalities are related to long-term dialysis treatment." Dujic et al. [19] also reported that patients on an everyday hemodialysis program for over 2 years had evidence of pulmonary diffusing capacity abnormalities.

By the way, we calculated the GFR in our study of our sample patients by the foremost preferred equation to nephrologists: CKD-EPI Creatinine 2009 equation which estimated from serum creatinine, age, sex, and race [6]. No previous study assessed the connection between CRF and voice, performed on sample sizes like our study including 54 subjects with CRF and 56 normal. This idea gives our study more priority and reliability.

There was a statistically direct correlation between diabetes and hypertension because the causes of CRF and dysphonia, and there was a statistically direct correlation between diabetes and hypertension because of the causes of CRF and the total VHI score. This could be explained by diabetes and hypertension themselves have effects on the voice and larynx, so, in our study overlapping occurred by hypertension and chronic kidney failure or by diabetes and chronic kidney failure. However, some proportion of our patients has hypertension and diabetes as causes of kidney failure. Barry and Materson [20] found that the majority of medications taken in hypertension affect the voice due to their drying effect on the protective mucosal layer covering the vocal folds which cause difficult phonation.

\section{Conclusion}

The results of our study revealed that subjects with chronic failure exhibit clinical evidence of voice disorders. The voice problems can vary between CRF patients depending on the duration of hemodialysis and the leading causes of CRF.

\section{Appendix}

Arabic version of the Voice Handicap Index (VHI)

\section{Appendix JJJ}

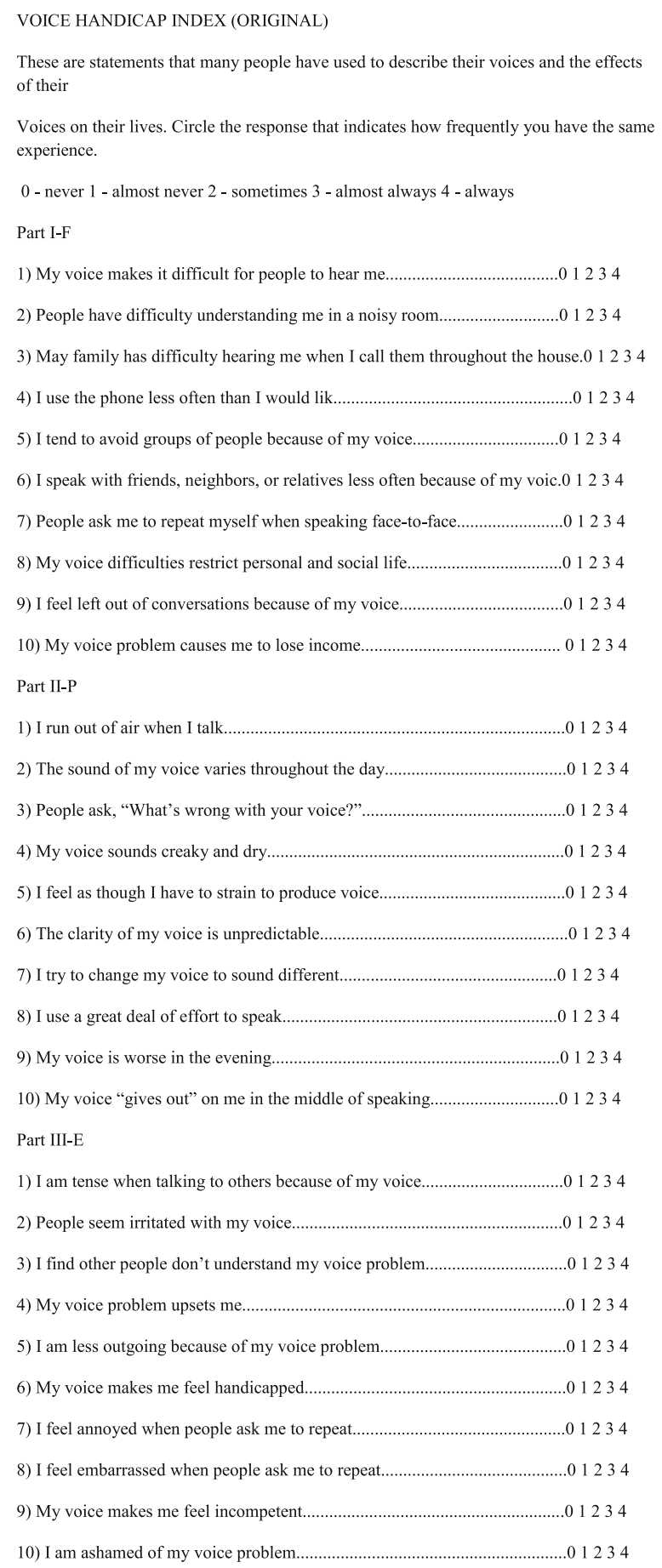

The Voice Handicap Index (VHI): Development and Validation

Barbara H. Jacobson, Alex Johnson, Cynthia Grywalski, Alice Silbergleit, Gary Jacobson, and Michael S (1997): Benninger .American Journal of Speech-Language Pathology, Vol 6(3), 66-70, Words in bold and underlined are those that are further explained in the Arabic version. 


\section{Abbreviations}

HD: Hemodialysis; ESRD: End-stage renal disease; CRF: Chronic kidney failure; VHI: Voice Handicap Index; GFR: Glomerular filtration rate; CR: Serum creatinine; SPSS: Statistical Package for the Social Sciences

\section{Acknowledgements}

Not applicable

\section{Authors' contributions}

$E A Z, O E$, and ZK conceived of the presented idea. EAZ, HM, and OE designed research. ZK, EAZ, and performed the analytic calculations and performed the numerical simulations. ZK, OE, and EAZ conducted review and editing. EAZ and wrote the paper. All authors read and approved the final manuscript.

\section{Funding}

No funding was obtained for this study.

\section{Availability of data and materials}

All data generated or analyzed during this study are included in this published article [and its supplementary information files].

\section{Ethics approval and consent to participate}

This study was approved by the ethics committee of Minia university Hospital, Faculty of medicine Egypt and the Egyptian Network of Research Ethics Committees ENREC. (Faculty Council Approval Date: 23rd January 2017). All patients participate in this research gave a written consent to participate within this research.

\section{Consent for publication}

Not applicable

\section{Competing interests}

The authors declare that they have no competing interests.

Received: 17 June 2020 Accepted: 5 October 2020

Published online: 25 November 2020

\section{References}

1. Longo DL, Fauci AL, Kasper DL, Hauser SL, Jamson JL (2011) Principles of internal medicine, 18th edn. MC Graw-hill, New York, pp 22-28

2. Ori Y, Sabo R, Binder Y (2006) Effect of hemodialysis on the thickness of vocal folds: a possible explanation for post dialysis hoarseness. Nephron Clin Pract. 103(4):144-148

3. Belafsky PC, Pastma GN, Kaufman JA (2001) The validity and reliability of reflux finding score (RFS). Laryngoscope 111:1313-1317

4. Kotby MN (1995) The accent method of voice therapy, vol 50. Singular Publishing Group, San Diego, pp 146-164

5. Jacobson BH, Johansen A, Grywaski C, Newman CW (1997) The voice Handicap Index (VHI): development and validation. Am J Speech Lang Pathol 630:66-70

6. Levey AS, Stevens LA, Schmid CH (2009) A new equation to estimate glomerular filtration rate. Ann intern Med 150(9):604-612

7. Mckinney JC (1994) The diagnosis and correction of vocal faults. Genevox Music Group, Nashville

8. Bossola M, Vulpio C, Tazza L (2011) Fatigue in chronic dialysis patients. SeminDial 24:550-555

9. Venkata RK, Kumar S, Krishna RP, Kumar SB, Padmanabhan S (2007) Tuberculosis in chronic kidney disease. Clin Nephrol 67:217-220

10. Kumar RB, Bhat JS (2010) Voice in chronic renal failure. J Voice 24:690-693

11. Hemler RJ, Wieneke GH, Lebacq JLD, Ejonckere PH (1999) Mechanical properties of excised vocal fold mucosa in high and low relative air humidity. Paper presented at the 2 nd Interna-tional Conference on Voice Physiology and Biomechanics, Berlin

12. Chen CW, Lee CH, Chang HY, Hsiue TR, Sung JM, Huang JJ (1998) Respiratory mechanics before and after hemodialysis in mechanically ventilated patients. J Formos Me Assoc 97:271-277
13. Nesic M, Veljkovic S, Obrenovic J, Cekic S, Velickovic D, Radenkovic M (1996) Voice frequencies in patients treated with chronic hemodialysis. SrpArhCelok Lek 124(1):99-101

14. Eman SH (2014) Effect of chronic renal failure on voice: an acoustic and aerodynamic analysis. J Voice 30(1):53-57

15. Maria C, Kenneth $O$ (2009) Effects of hydration on voice acoustics. J Voice 36:142-148

16. Unver S, Umit H, Kenan E, Adem S, Fatma C (2015) Objective analysis of voice changes in a hemodialysis session and its correlation with ultrafiltration. J Voice 37(2):267-272

17. Pierson D (2006) Respiratory considerations in the patients with renal failure. Respir Care 51(2):61-67

18. Moinard J, Guenard H (1993) Membrane diffusion on the lungs in patients with chronic renal failure. Eur Respir 6:225-230

19. Dujic Z, Tocilj J, Ljutic D, Eterovic D (1991) Effects of hemodialysis and anemia on pulmonary diffusing capacity, membrane diffusing capacity and capillary blood volume in uremic patients. Respiration 58:277-228

20. Barry J, Materson MD (1992) Adverse effects of angiotensin-converting enzyme inhibitors in antihypertensive therapy with focus on quinapril. Am J Cardiol 69(10):46-53

\section{Publisher's Note}

Springer Nature remains neutral with regard to jurisdictional claims in published maps and institutional affiliations.

\section{Submit your manuscript to a SpringerOpen ${ }^{\circ}$ journal and benefit from:}

- Convenient online submission

- Rigorous peer review

- Open access: articles freely available online

- High visibility within the field

- Retaining the copyright to your article

Submit your next manuscript at $\boldsymbol{\nabla}$ springeropen.com 